\title{
Lumbar Epidural Steroid Injection for Painful Spasticity in Cervical Spinal Cord Injury: A Case Report
}

\author{
Hyun Bang, MD, Seong Min Chun, MD, Hee Won Park, MD, Moon Sulk Bang, MD, Keewon Kim, MD
}

Department of Rehabilitation Medicine, Seoul National University College of Medicine, Seoul, Korea

We report a case of a 53-year-old male with traumatic cervical spinal cord injury (SCI). He could not maintain a standing position because of painful spasticity in his lower limbs. A magnetic resonance imaging and electromyography indicated chronic lumbosacral radiculopathy, explaining his chronic low back pain before the injury. For diagnostic as well as therapeutic purposes, transforaminal epidural steroid injection (ESI) to the right L5 root was performed. After the intervention, the spasticity decreased and his ambulatory function improved. This case illustrates that lumbar radiculopathy concomitant with a cervical SCI can produce severe spasticity and it can be dramatically improved by ESI.

Keywords Spinal cord injuries, Muscle spasticity, Radiculopathy

\section{INTRODUCTION}

Spasticity is a common complication of spinal cord injury (SCI), experienced by up to $80 \%$ of SCI patients [1]. Although spasticity may help patients stand or move and enhance circulation in the lower limbs, it may also cause functional deterioration, joint contracture, ulcers, and pain. To reduce spasticity, a combination of various treatments, including physiotherapy, medication, botulinum toxin injection, and intrathecal infusion of baclofen, can

Received August 19, 2014; Accepted September 18, 2014

Corresponding author: Keewon Kim

Department of Rehabilitation Medicine, Seoul National University College of Medicine, 101 Daehang-ro, Jongno-gu, Seoul 110-744, Korea

Tel: +82-2-2072-2619, Fax: +82-2-743-7473, E-mail: keewonkimm.d@ gmail.com

(c) This is an open-access article distributed under the terms of the Creative Commons Attribution Non-Commercial License (http://creativecommons. org/licenses/by-nc/4.0) which permits unrestricted noncommercial use, distribution, and reproduction in any medium, provided the original work is properly cited.

Copyright (C) 2015 by Korean Academy of Rehabilitation Medicine be applied [2].

The pathophysiology of spasticity after SCI is still unclear. It is generally explained as hyper-excitability of the spinal reflex. Spasticity consists of intrinsic and extrinsic components [1]. Intrinsic spasticity, which can be tonic or phasic, refers to exaggerated stretch reflexes unrelated to external stimuli. Extrinsic components appear as involuntary muscle spasms in response to a perceived noxious stimulus below the injury level. Spasticity can be triggered or aggravated by various peripheral nociception in the skin, muscle, or joints [2]. Therefore, in clinical practice, appropriate management of spasticity requires the investigation of aggravating factors and treatment if necessary.

However, causality between possible noxious stimulus and spasticity is not easily confirmed. In most of cases, the extrinsic component of spasticity cannot be differentiated from the intrinsic component merely by physical evaluation; only after eliminating nociceptive input can 
the extrinsic component be identified. Without a high index of suspicion, spasticity of the lower extremities can be hastily concluded to be the inevitable sequelae of SCI.

To the authors' knowledge, there are no existing reports suggesting lumbosacral radiculopathy as a triggering or aggravating factor of spasticity in cervical SCI patients. In this report, we present a patient with incomplete tetraplegia whose severe pain and restricted mobility due to lower limb spasticity decreased significantly after lumbar epidural steroid injection (ESI) and discuss relevant clinical implications.

\section{CASE REPORT}

A 53-year-old man fell down in a drunken state. He had cervical SCI and underwent subtotal laminectomy at C3 and $\mathrm{C} 4$ levels. He developed incomplete tetraplegia and was transferred to the rehabilitation unit at a tertiary university hospital. At the time of hospitalization, his neurological level of injury was C5 and the American Spinal Injury Association Impairment Scale (AIS) was grade C; motor level C5/C5, sensory level T4/T4, motor score $21 / 27$, sensory score 78 for light touch and 112 for pin prick. He had an infected sacral sore. Local flap surgery was performed 6 months after the injury. He was then transferred to a rehabilitation hospital and continued physiotherapy for 6 months.

One year after the injury, he was admitted again to the university hospital. At the time, the AIS was grade D and the neurological level of injury remained at C5; motor level C5/C5, sensory level undetermined, motor score $32 / 37$, sensory score 112 for light touch and pin prick in total. He was still unable to maintain standing balance for one minute due to severe pain and spasticity of the lower limbs, especially on the right side. Knee flexor spasticity was grade $3 / 1+$ (right/left) and right knee extensor spasticity was grade 3 by the Modified Ashworth Scale (MAS). Although he was taking baclofen three times a day (1010-30 mg) and $2 \mathrm{mg}$ diazepam once a day, it was of little effect. Painful spasms in the hamstrings occurred periodically about once an hour, which severely interrupted physiotherapy and even disturbed his sleep. In addition, he was suffering from pain that radiated from the buttock to the posterolateral calf and medial foot on the right side. He described the character as 'stiff, aching, and tingling' and the intensity as 9 out of 10 on the numeric rating scale (NRS). The pain was aggravated by sitting for about 5 minutes or longer and was relieved in a supine
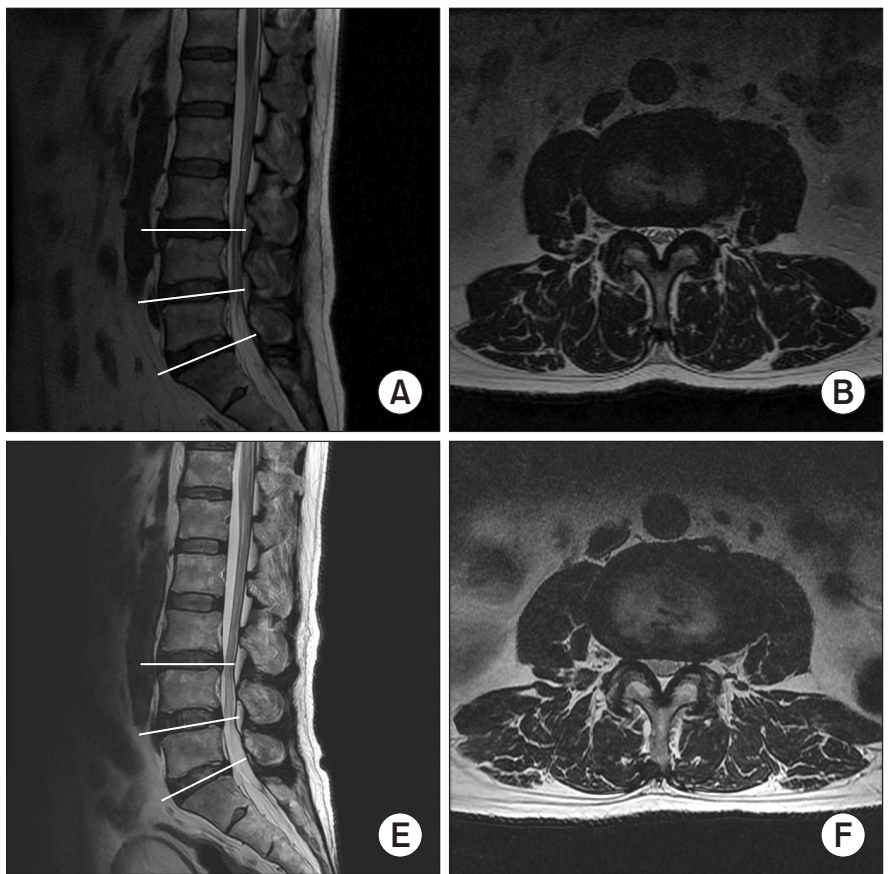
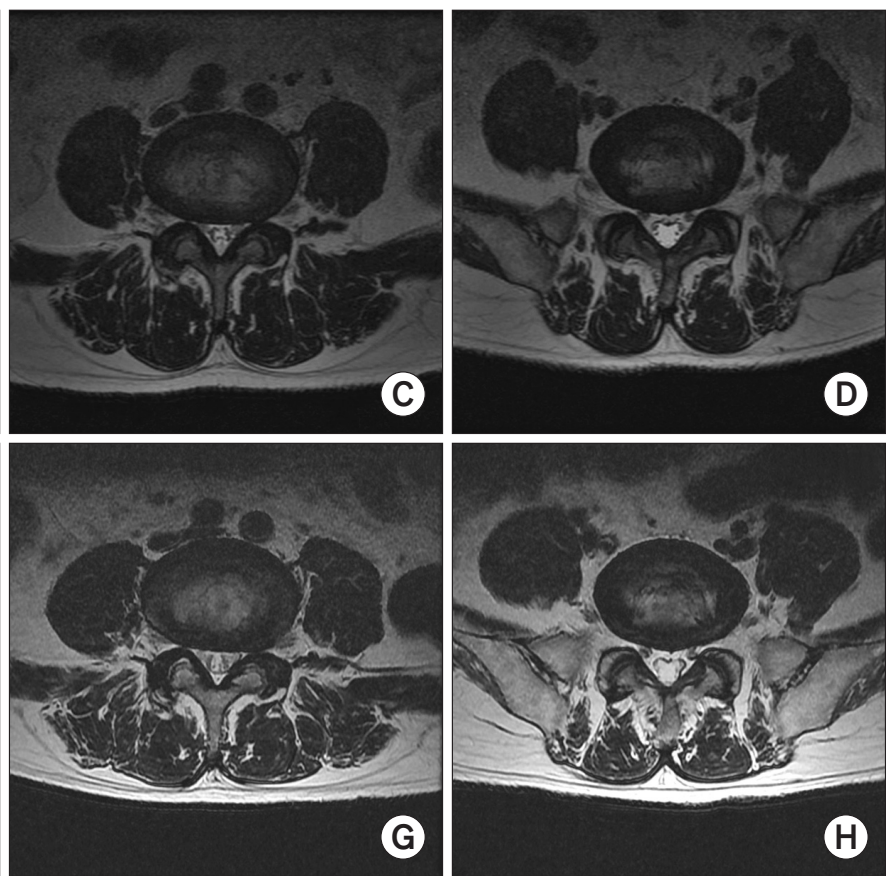

Fig. 1. T2-weighted magnetic resonance imaging of the lumbar spine. (A-D) About 1 year before the injury. (A) Midsagittal view. (B) Axial view at L3/4 level. (C) Axial view at L4/5 level. (D) Axial view at L5/S1 level. (E-H) After the cord injury. (E) Mid-sagittal view. (F) Axial view at L3/4 level. (G) Axial view at L4/5 level. (H) Axial view at L5/S1 level. 


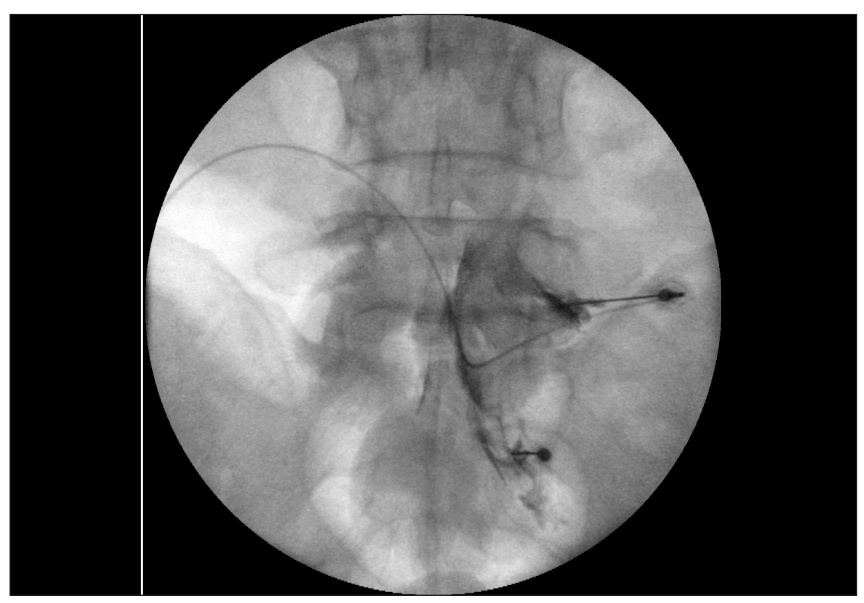

Fig. 2. Right L5 transforaminal epidural steroid injection under fluoroscopic guidance. Injection level was determined by symptom provocation with $0.9 \%$ normal saline at L5 and S1 levels.

position. The pain did not respond to $300 \mathrm{mg}$ gabapentin three times a day. His activities of daily living were generally restricted because he could not maintain sitting due to pain.

According to his past medical history, he had experienced a chronic intermittent low back pain radiating to his right thigh and calf before the cord injury. He stated that the previous symptoms were tolerable with conservative management including physiotherapy and oral medication. Although its severity was milder than his present symptoms and it was not accompanied by spasticity, the past low back pain was similar to the present in that it was aggravated by walking and long sitting. He had a magnetic resonance imaging (MRI) of the lumbar spine before the cord injury. It demonstrated disc bulging at L3/4 and L4/5 levels (Fig. 1A-D).

Based on the suspicion that a lumbosacral radiculopathy might be associated with his current spasticity and pain of the lower limbs, a follow-up MRI was performed. The MRI showed no notable changes in the bulging discs at the L3/4 and L4/5 (Fig. 1E-H). The patient underwent an electrodiagnostic study for chronic right L5 and S1 radiculopathy with bilateral peroneal neuropathy. For diagnostic as well as therapeutic purposes, transforaminal ESI was planned. Considering the dermatome of symptoms and the results of the electrodiagnostic study, transforaminal ESI was performed targeting the right L5 root under fluoroscopic guidance after symptom provocation with $0.9 \%$ normal saline at L5 and S1 levels (Fig. 2). The drug injected was a mixture of triamcinolone acetonide $40 \mathrm{mg}, 0.9 \%$ normal saline $3 \mathrm{~mL}$, and ropivacaine $\mathrm{HCl}$ $0.75 \% 1 \mathrm{~mL}$.

Immediately after the intervention, the painful spasticity of the right lower limb subsided. The next day, the spasticity of the knee flexor and extensor decreased from 3 to $1+$ (MAS) and the pain intensity decreased from $9 / 10$ to $3 / 10$ (NRS). The distribution of the radiating pain shrunk from the entire right lower limb to the right buttock area. The periodic spasm in the right hamstring disappeared and sleep hygiene improved accordingly. $\mathrm{He}$ could perform gait training actively with improvements in spasticity and pain. Three weeks after the intervention, he could walk with a walker for about $100 \mathrm{~m}$ without rest. He was then discharged and continued rehabilitation at an outpatient clinic. His improved pain and spasticity was maintained at the follow-up visit one year after the single intervention.

\section{DISCUSSION}

Spasticity after SCI often causes joint contractures, pressure sores, or pain, thereby compromising functionality and quality of life. Thus, spasticity should be diligently diagnosed and appropriately managed. In this case, spasticity with pain in the lower limbs had precluded the patient from gait training. However, it was dramatically alleviated after lumbar transforaminal ESI and his gait improved accordingly. To the authors' knowledge, this is the first report on the effect of lumbar ESI for painful spasticity of the lower limbs in a cervical SCI patient.

Spasticity can be triggered or aggravated in response to any noxious stimulus below the injury level [2]. We assume that lumbosacral radiculopathy was an extrinsic stimulus in this case. Thus, the symptoms were unusual and were more severe than other lumbosacral radiculopathies and therefore, ESI at the lumbar spine could drastically relieve the pain and spasticity. This explanation may be supported by the observation that spasticity was aggravated when sitting or standing, which are typical aggravating postures for low back pain by lumbosacral radiculopathy. In addition, his spasticity was asymmetric; the right side was much more severe than the left side. Usually, spasticity in SCI patients is symmetric whereas 
radicular pain from the lumbar level is asymmetric or unilateral. Additionally, in needle electromyography, high-amplitude and polyphasic motor unit action potentials were more frequently observed in the muscles of the right lower limb.

However, the causal relation of lumbar radiculopathy with spasticity was not evident. Although the patient experienced painful spasticity several months after the injury, there were no definite changes in the lumbar spine MRI compared with the MRI before the injury. While the symptoms were concentrated on the right side, spondylosis, disc herniation, or foraminal stenosis on the MRI did not show definite side-to-side differences. In addition, the electrophysiologic study did not reveal any ongoing or recent disease activity. The patient experienced breaking-through pain even while sleeping, which is rare in mechanical low back pain. Considering these points, L5 radiculopathy may not have been an aggravating factor for his clinical symptoms. Nonetheless, the fact that the spasticity improved remarkably after the lumbar spine intervention strongly advocated a causal relation.

There are a few factors that can explain concomitant degeneration of the lumbar spine in cervical spinal cord injury patients. First, multiple tandem lesions of the spine can exist in the cervical and lumbar spine simultaneously [3-5]. And the risk of traumatic SCI is much higher in patients with cervical spinal stenosis than in those without it [6]; patients with degeneration of the cervical spine can develop cervical SCI by minor trauma $[7,8]$. Thus, a patient with cervical SCI due to minor trauma would have had a higher incidence of lumbar spondylosis prior to the injury. The patient in this report also had a history of chronic low back pain with degenerative changes. Second, muscle weakness and impaired proprioception may predispose SCI patients to poor posture such as kyphosis or hyper-lordosis. Especially, weakness of the core muscles can directly undermine spinal stability and thereby cause low back pain $[9,10]$.

However, in cervical SCI patients, lumbosacral radiculopathy is not readily diagnosed without a high index of suspicion. Changes in neurophysiology after SCI can make the clinical presentation atypical in these patients. Patients may poorly localize the pain due to impaired sensation; aggravating factors may be different due to the change of motor function; and symptoms may manifest as spasticity as in this case. We recommend the consider- ation of lumbosacral radiculopathy as a cause of intractable lower extremity spasticity developed in higher level SCI patients. If suspected, ESI can be selected for a diagnostic as well as therapeutic approach.

To conclude, in this case with cervical SCI, lower limb spasticity and pain was dramatically reduced after ESI with functional improvement. Lumbosacral radiculopathy in SCI patients may not be readily recognized but can be a trigger for painful spasticity. We recommend the election of ESI if it is suspected.

\section{CONFLICT OF INTEREST}

No potential conflict of interest relevant to this article was reported.

\section{REFERENCES}

1. Adams MM, Hicks AL. Spasticity after spinal cord injury. Spinal Cord 2005;43:577-86.

2. Rekand T, Hagen EM, Gronning M. Spasticity following spinal cord injury. Tidsskr Nor Laegeforen 2012; 132:970-3.

3. Han IH, Suh SH, Kuh SU, Chin DK, Kim KS. Types and prevalence of coexisting spine lesions on whole spine sagittal MR images in surgical degenerative spinal diseases. Yonsei Med J 2010;51:414-20.

4. LaBan MM, Green ML. Concurrent (tandem) cervical and lumbar spinal stenosis: a 10-yr review of 54 hospitalized patients. Am J Phys Med Rehabil 2004;83:18790.

5. Aydogan M, Ozturk C, Mirzanli C, Karatoprak O, Tezer M, Hamzaoglu A. Treatment approach in tandem (concurrent) cervical and lumbar spinal stenosis. Acta Orthop Belg 2007;73:234-7.

6. Takao T, Morishita Y, Okada S, Maeda T, Katoh F, Ueta $\mathrm{T}$, et al. Clinical relationship between cervical spinal canal stenosis and traumatic cervical spinal cord injury without major fracture or dislocation. Eur Spine J 2013;22:2228-31.

7. Nakae R, Onda H, Yokobori S, Araki T, Fuse A, Toda $S$, et al. Clinical analysis of spinal cord injury with or without cervical ossification of the posterior longitudinal ligament, spondylosis, and canal stenosis in elderly head injury patients. Neurol Med Chir (Tokyo) 2010;50:461-5. 
8. Regenbogen VS, Rogers LF, Atlas SW, Kim KS. Cervical spinal cord injuries in patients with cervical spondylosis. AJR Am J Roentgenol 1986;146:277-84.

9. Akuthota V, Nadler SF. Core strengthening. Arch Phys
Med Rehabil 2004;85(3 Suppl 1):S86-92.

10. Hodges PW. Core stability exercise in chronic low back pain. Orthop Clin North Am 2003;34:245-54. 